\title{
Dual-Channel Forward-Looking SAR Micro-Motion Targets Detection
}

\author{
Chen Lei ${ }^{1}$, Chen Dian-ren ${ }^{1}$, Sha Sha ${ }^{2}$, Liu Ying ${ }^{1} \&$ Li Xing-guang ${ }^{1}$ \\ ${ }^{1}$ College of Electronic Information and Engineering, Changchun University of Science and Technology, \\ Changchun, China \\ ${ }^{2}$ Jilin Dongguang Aowei automobile brake system Co. Ltd, Changchun, China \\ Correspondence: Lei Chen, Changchun University of Science and Technology, Changchun, China. Tel: \\ 86-138-4304-7402. E-mail: chenlei511@126.com
}

Received: October 16, 2014

Accepted: October 20, $2014 \quad$ Online Published: January 10, 2015

doi:10.5539/mas.v9n2p185

URL: http://dx.doi.org/10.5539/mas.v9n2p185

\begin{abstract}
It is aimed at the problem of forward-looking SAR micro-motion target detection in this paper. The traditional dual-channel SAR DPCA cancellation principle is analyzed and a dual-channel DPCA model of forward-looking SAR is proposed, the amplitude and phase characters of the signal after DPCA cancellation is studied, the distance domain of the micro-motion target is determined, then the micro-motion target detection is probability under different echo SNR with Monte-Carlo Operation, when the echo SNR is bigger than $-5 \mathrm{~dB}$, and the target detection probability is greater than $90 \%$.
\end{abstract}

Keywords: forward-looking SAR, micro-motion, target detection, dual-channel, DPCA, detection probability

\section{Introduction}

The dual-channel SAR ground moving target detection algorithm include: Space Time Sdaptive Processing (STAP) technique ${ }^{[1]-[3]}$, Along Track Interferometry (ATI) ${ }^{[4]-[5]}$, displaced phase center antenna (DPCA) clutter cancellation ${ }^{[6]}$ etc.. The traditional dual-channel side-looking SAR target detection system require two sub channels, it increases the complexity of radar front-end, because of the special geometric structure of forward-looking SAR, the dual-channel mode could be simple realized through changing the antenna transmit and receiving order on the premise of not increasing the hardware resources. The dual-channel DPCA micro-motion dection method is worthy of study in the forward-looking SAR system.

\section{Dual-Channel DPCA Cancellation Principle}

Because of the simple principle, DPCA clutters reject is widely used in SAR moving target detection system.The stationary target echo phase of different apertures is consistent but the moving target echo phase is inconsistent, so the stationary target is preserved after clutter cancellation, the clutter cancellation formula could be expressed as:

$$
\operatorname{DPCA}(t)=s_{1}(t)-s_{2}(t)
$$

In which, $s_{1}(t), s_{2}(t)$ are the echoes of the channels.

In the side-looking SAR DPCA system, the subaperture is composed of different sub antennas, the physical structure of traditional DPCA platform is shown in Figure 1. In order to keep sub aperture phase center consistency, the flight velocity equals the distance between the antennas' divided transmitted pulse period.

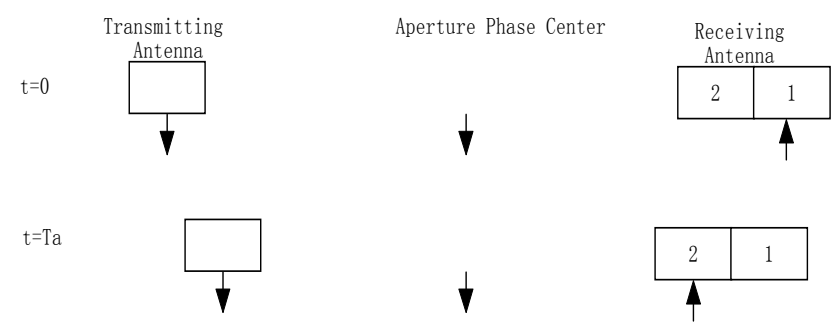

Figure 1. dual-channel DPCA cancellation principle 


\section{Dual-Channel Forward-Looking SAR DPCA Principle}

There are a plurality of antenna array in the azimuth of forward-looking SAR, so it does not need to modify the radar front-end hardware when the DPCA technology is applied to the forward looking SRA.The antennas could be divided into two groups and the consistency of the phase center need to be considered.The send and receive sequence as shown in figure 2 could meet the requirements.

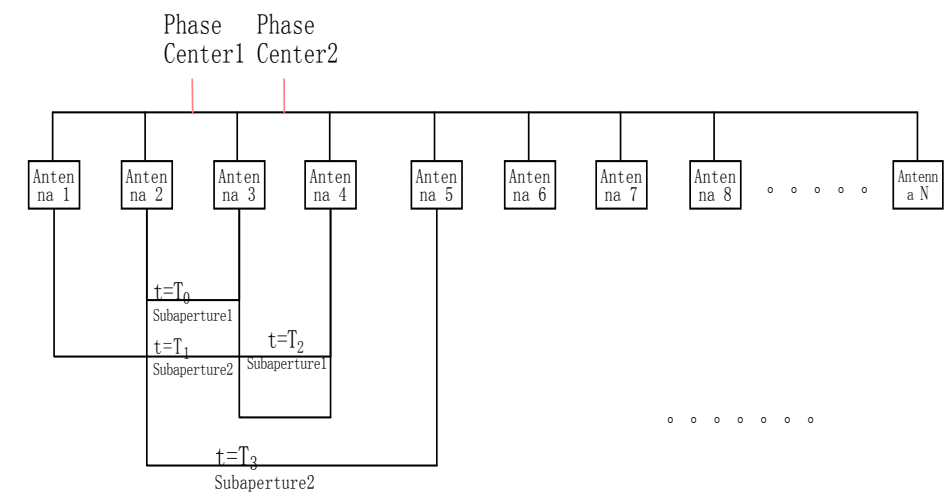

Figure 2. forward-looking SAR DPCA working mode

As shown in Figure 2, the antenna arrays of forward-looking SAR DPCA working mode are grouped into: (Antenna 1 transmit, Antenna 4 receive), (Antenna 1 transmit, Antenna 4 receive) (Antenna 1 transmit, Antenna 4 receive) (Antenna 1 transmit, Antenna 4 receive) etc.. when $\mathrm{t}=\mathrm{T}_{0}$, Antenna 1 transmit, Antenna 4 receive, forming subaperture 1, when $\mathrm{t}=\mathrm{T}_{1}$, Antenna 2 transmit, Antenna 3 receive, forming Subaperture 2, when $\mathrm{t}=\mathrm{T}_{2}$, Antenna 2 transmit, Antenna 5 receive, forming Subaperture 1, when $\mathrm{t}=\mathrm{T}_{3}$, Antenna 3 transmit, Antenna 4 receive, forming Subaperture 2, and so on. Figure 2 clearly shows that the phase centers of subaperture 1 and subaperture 2 is consistent.

\section{Dual-Channel Forward-Looking SAR Micro-Motion Target DPCA Model}

As shown in Figure2, the distance between antennas could be expressed as:

$$
\mathrm{d}=\mathrm{mT}_{\mathrm{a}}
$$

The equivalent phase center of the subapertures is half of d,its working mode could be approximated as two antennas transmit and receive independently. At any time of t,the slant-range between forward looking targets and radar antennas can be expressed as :

$$
R_{1}(t) \approx \sqrt{\left(V_{a} t-x_{0}\right)^{2}+y_{0}^{2}+H^{2}}+r \cos \left(\omega t+\phi_{0}\right)
$$

Then at any time of $t$, the slant-range of equivalent aperture and radar forward looking target can be expressed as:

$$
R_{2}(t) \approx \sqrt{\left(-d+V_{a} t-x_{0}\right)^{2}+y_{0}^{2}+H^{2}}+r \cos \left(\omega t+\phi_{0}\right)
$$

Register the formula (4) and obtain:

$$
R_{2 \text { reg. }}(t)=R_{2}(t+m T) \approx \sqrt{\left(V_{a} t-x_{0}\right)^{2}+y_{0}^{2}+H^{2}}+r \cos \left[\omega(t+m T)+\phi_{0}\right]
$$

According to the above assumption of parameters of the radar, radar echoes of subaperture 1 and subaperture 2 can be expressed as:

$$
\begin{array}{r}
S_{1}(\tau, t)=A \cdot \operatorname{Sinc}\left[B\left(\tau-\frac{2 R_{1}(t)}{c}\right)\right] \exp \left[-j \frac{4 \pi R_{1}(t)}{\lambda}\right] \\
S_{2}(\tau, t)=A \cdot \operatorname{Sinc}\left[B\left(\tau-\frac{2 R_{2 \text { reg }}(t)}{c}\right)\right] \exp \left[-j \frac{4 \pi R_{\text {2reg }}(t)}{\lambda}\right]
\end{array}
$$

The expression for the azimuth echo can be expressed as: 


$$
\begin{aligned}
& s_{1}(t)=A_{0} \exp \left[-j \frac{4 \pi R_{1}(t)}{\lambda}\right] \\
& s_{2}(t)=A_{0} \exp \left[-j \frac{4 \pi R_{2 \text { reg }}(t)}{\lambda}\right]
\end{aligned}
$$

\section{DPCA Cancellation Signal Characteristics}

The ideas of DPCA is ${ }^{[9]}$ : make each sub-aperture echo cancellate and eliminate the effects of stationary target and background.To detect the micro-motion targets, we assume:

$$
\begin{gathered}
\phi_{1}=-\frac{\Delta \pi R_{\mathrm{l}^{\prime}}(t)}{\lambda} \\
\phi_{2} \stackrel{\Delta}{=}-\frac{4 \pi R_{2 \mathrm{reg}}(t)}{\lambda}
\end{gathered}
$$

In which $R_{1 \mathrm{o}}(t)$ and $R_{2 \text { reg。 }}(t)$ can be expressed as:

$$
\begin{gathered}
R_{1_{\circ}}(t)=-\frac{\lambda \phi_{1}}{4 \pi} \\
R_{2 \text { reg。 }}(t)=-\frac{\phi_{2} \lambda}{4 \pi}
\end{gathered}
$$

With the principle of DPCA:

$$
\begin{aligned}
D P C A(t) & =s_{1}(t)-s_{2}(t) \\
& =A \cdot\left[\exp \left(j \phi_{1}\right)-\exp \left(j \phi_{2}\right)\right] \\
& =A \cdot \sin \left(\frac{\phi_{1}-\phi_{2}}{2}\right) \exp \left(j \frac{\phi_{1}+\phi_{2}+\pi}{2}\right)
\end{aligned}
$$

With formula (12) and formula (13), formula (14) could be expressed as:

$$
D P C A(t)=2 A_{0} \sin \left[\frac{2 \pi}{\lambda}\left(R_{2 \text { reg }}(t)-R_{1}(t)\right)\right] \exp \left\{j\left[-\frac{2 \pi}{\lambda}\left(R_{1}(t)+R_{2 \text { reg }}(t)\right)+\frac{\pi}{2}\right]\right\}
$$

After taking formula (3) (5) (6) into the equation (15), its absolute value is:

$$
\begin{aligned}
|D P C A(t)|=\left|2 A \cdot \sin \left[\frac{2 \pi}{\lambda_{c}}\left(R_{2 \text { reg. }}(t)-R_{1}(t)\right)\right]\right| & \approx\left|2 A \cdot \sin \left\{\frac{2 \pi}{\lambda} r\left\{\cos \left[\omega(t+m T)+\phi_{0}\right]-\cos \left(\omega t+\phi_{0}\right)\right\}\right\}\right| \\
& =\left|2 A \cdot \sin \left[-\frac{4 \pi}{\lambda} r \sin \left(\frac{\omega d}{2 V}\right) \sin \left(\omega t+\frac{\omega d}{2 V}+\phi_{0}\right)\right]\right|
\end{aligned}
$$

The Taylor series expansion of formula (16) can be expressed as:

$$
|D P C A(t)| \approx\left|\frac{8 \pi}{\lambda} A \cdot \gamma \cdot \sin \left(\frac{\omega d}{2 V_{\alpha}}\right) \cdot \sin \left(\omega t+\frac{\omega d}{2 V_{\alpha}}+\phi_{0}\right)\right|
$$

Because the forward-looking SAR imaging process time is very short, the value of $t$ is small, also, because the target belongs to the micro-motion category, the value of $\omega t$ is very small, then we can assume that $\sin \theta \approx \theta$. then:

$$
\left|-\frac{4 \pi}{\lambda_{c}} \gamma_{m} \sin \left(\frac{\omega d}{2 V_{\alpha}}\right) \sin \left(\omega t+\frac{\omega d}{2 V_{\alpha}}+\phi_{0}\right)\right| \leq \frac{2 \pi \gamma_{m} \omega d}{\lambda_{c V_{\alpha}}} \leq \frac{1}{18} \pi
$$

Therefore, the conditional expression of equation 17 can be written as: 


$$
\gamma_{m} \omega \geq \frac{\lambda_{c} V_{\alpha}}{36 d}
$$

Where $\gamma_{m} \omega$ is instantaneous line speed which is caused by the target micro-motion angular velocity,for the formula (19), the motion parameters of micro-motion target studied in this paper can be met.By the formula (17), after DPCA clutter cancellation the amplitude conversion of micro-motion target forward looking SAR echo signal may be represented as a sinusoidal function formed of $(|A \sin (\omega t+\phi)|)$, where the value of $\mathrm{A}$ is affected by the target micro-motion amplitude and frequency.

According to equation (16), the phase characteristics of DPCA cancellation signal could be expressed as:

$$
\begin{aligned}
\arg [D P C A(t)]= & -\frac{2 \pi}{\lambda c}\left(R_{1}(t)+R_{2 r e g}(t)\right)+\frac{\pi}{2} \\
\approx & -\frac{2 \pi}{\lambda c}\left\{2 \sqrt{\left(V_{a} t-X_{n c}\right)^{2}+Y_{n c}^{2}+H^{2}}\right. \\
& \left.+r_{m}\left\{\cos \left(\omega t+\phi_{0}\right)+\cos \left[\omega\left(t+\frac{d}{V_{a}}\right)+\phi_{0}\right]\right\}\right\}+\frac{\pi}{2} \\
= & -\frac{4 \pi}{\lambda_{c}}\left[\sqrt{\left(V_{a} t-X_{n c}\right)^{2}+Y_{n c}^{2}+H^{2}}+r_{m} \cos \left(\frac{\omega d}{2 V_{a}}\right) \cos \left(\omega t+\frac{\omega d}{2 V_{a}}+\phi_{0}\right)\right]+\frac{\pi}{2}
\end{aligned}
$$

Formula (20) shows that, phase characteristics of the cancellation signal through DPCA also have a sinusoidal modulation characteristics, through the above analysis, both the amplitude and phase of the forward-looking SAR echo after cancellation through DPCA have a sinusoidal modulation characteristics ${ }^{[10]}$.

\section{The Simulation of Cancellation Signal through DPCA}

Suppose that there exists a micro-motion target and a stationary target in the imaging scene, in order to facilitate the analysis, the target detction process performance is simulated with MATLAB. Radar parameters is shown in Table 1; Target motion parameters is shown in Table 2:

Table 1. Radar parameters

\begin{tabular}{lcc}
\hline Parameters & Value & Unit \\
\hline Wavelength & 0.0033 & $\mathrm{~m}$ \\
Radar velocity & 300 & $\mathrm{~m} / \mathrm{s}$ \\
Frequency modulation rate & 100 & $\mathrm{MHz} / \mathrm{s}$ \\
The number of antenna array elements & 60 & \\
Pulse width & 1.5 & $\mu \mathrm{s}$ \\
Radar platform height & 1000 & $\mathrm{~m}$ \\
Pulse frequency & 10 & $\mathrm{KHz}$ \\
\hline
\end{tabular}

Table 2. Target motion parameters

\begin{tabular}{lccc}
\hline parameters & target 1 & Stationary target & unit \\
\hline micro-motion radius & 40 & 0 & $\mathrm{~m}$ \\
micro-motion angular velocity & $0.5 \pi$ & 0 & $\mathrm{rad} / \mathrm{s}$ \\
movement center coordinates & $(380,300)$ & $(500,300)$ & $\mathrm{m}$ \\
\hline
\end{tabular}

The simulation results are shown as Figure 3 and Figure 4, the raw data of sub-aperture 1 and sub-aperture 2 are shown as Figure 3(a)(b), the figure shows that we are unable to determine whether exists micro-motion target scene in the original signal.The imaging result of sub-aperture 1 is shown as Figure 3 (c), the imaging result of sub-aperture 2 is shown as Figure 3 (d), with Formula (19) and Formula (20), the parabola pixels appear in the image, it proved that there have micro-motion target in the sence. 


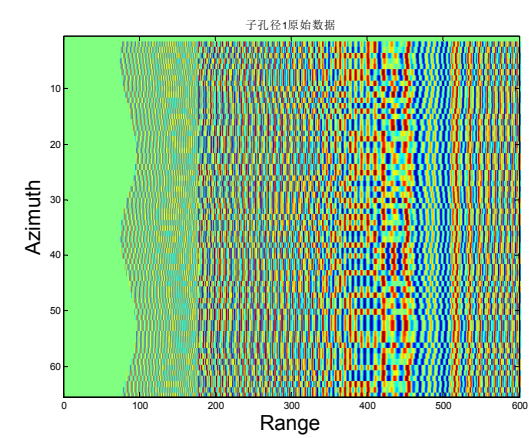

(a) raw data of sub-aperture 1

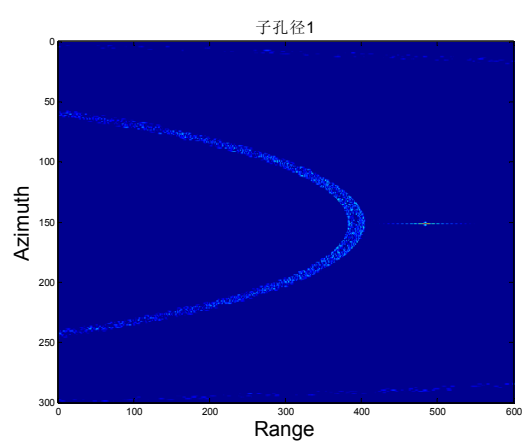

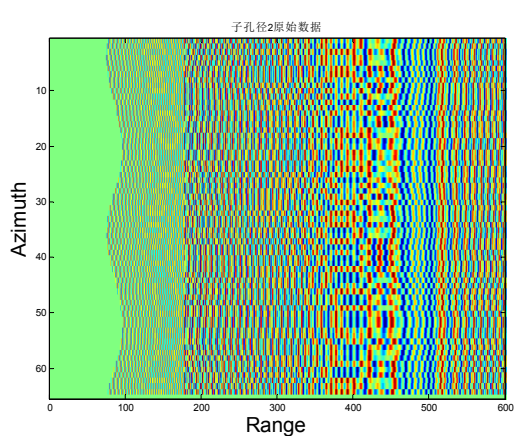

(b) raw data of sub-aperture 2

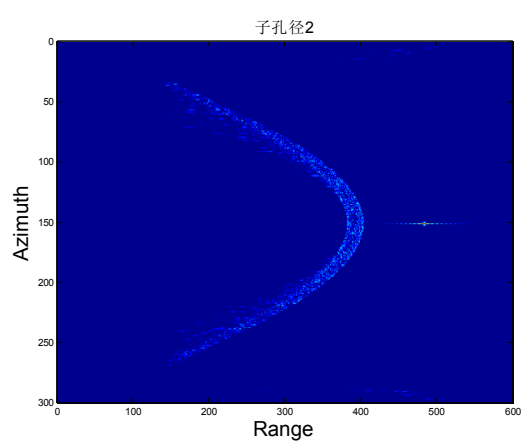

(c) imaging result of sub-aperture 1 (d)imaging result of sub-aperture 2

Figure 3. raw data and imaging results of sub-apertures

In Figure 4, micro-motion components have been preserved after cancellation through DPCA, two parabolas occurs in the image because that the radar carrier aircraft is in the different range coordinate of sub-aperture 1 and sub-aperture 2 imaging. We can choose radar carrier aircraft speed to compensate to solve this problem.

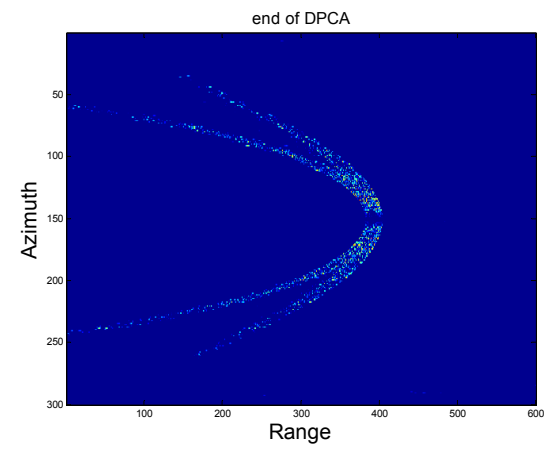

Figure 4. Results of cancellation signal through DPCA

Figure 5 (a) is the azimuth accumulated results of cancellation signal through DPCA, it can be clearly seen that in the image peak value appears in the coordinate of 380 , it is the micro-motion target range position located.In order to achieve the effect of automatic detection, we can set a threshold, the threshold value is $\kappa$ times of the standard deviation of the local data, the value of $\kappa$ determines the probability of false alarm of detection.In order to verify the detection results, calculate the target detection probability under different SNR with Monte-Carlo operation. The simulation results are shown in Figure 5(b): 


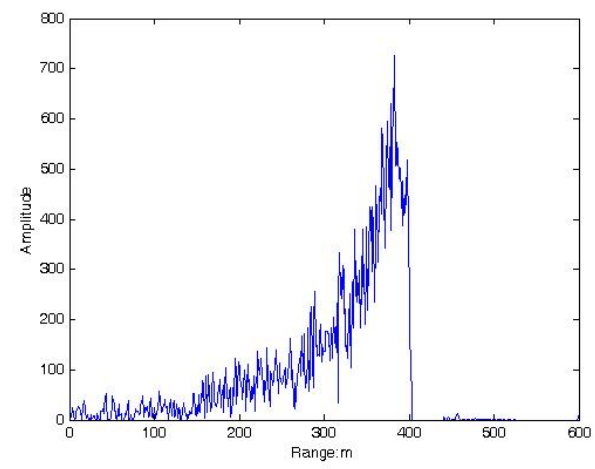

(a) accumulated results along the distance

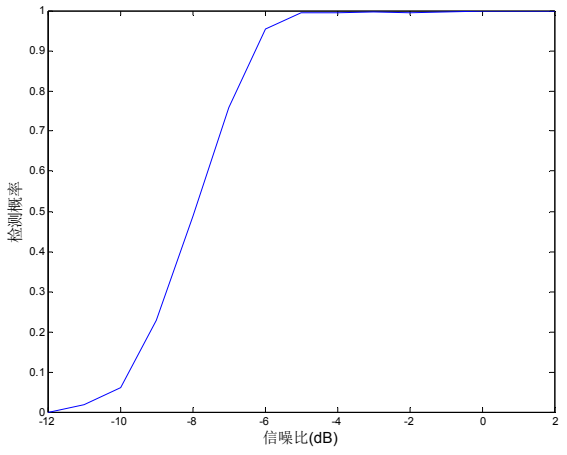

(b) the simulation results of Monte-Carlo

Figure 5. accumulated results along the distance

Figure 5 (b) shows that when the SNR of radar echo is greater than $-5 \mathrm{~dB}$, the micro-motion target detection probability reaches $95 \%$.

\section{Conclusion}

It is around the characters of the signal after DPCA cancellation,studied a micro-motion target dection mothed based on dual-channel forward-looking SAR DPCA in this paper.A kind of dual-channel forward-looking SAR working mode based on the special antenna transceiver array Structure is proposed. A echo mathematical model of the micro-motion target under this mode is deduced, the characters of the signal after DPCA cancellation is obtained, The simulation results show the effectiveness of the system.

\section{Reference}

Cerutti-Maori, D., \& Sikaneta, I. (2013). A Generalization of DPCA Processing for Multichannel SAR/GMTI Radars. IEEE Transactions on Geoscience and Remote Sensing, 51(1), 560-572. http://dx.doi.org/ 10.1109/TGRS.2012.2201260

Geroleo, F. G. (2012). Brandt-Pearce M. Detection and estimation of LFMCW radar signals. IEEE Transactions on Aerospace and Electronic Systems, 48(1), 405-418. http://dx.doi.org/10.1109/TAES.2012.6129644

Geroleo, F. G., \& Brandt, P. M. (2012). Detection and estimation of LFMCW radar signals.IEEE Transactions on Aerospace and Electronic Systems, 48(1), 405-418.

Jianbo, Y. (2012). Instrumentation and Measurement, IEEE Transactions on, 8(61), $2200-2211$. http://dx.doi.org/10.1109/TIM.2012.2184015

Misiurewicz, J., Kulpa, K., \& Czekala, Z. (1997). Analysis of Recorded Helicopter Echo. IEEE Radar, 97, Proceedings, 449-453. http://dx.doi.org/10.1049/cp:19971715

Weiguo, W., Lei, S., Xiaobo, L., \& Hongbo, L. (2013). Application of DPCA based stochastic filtering model and comparison of optimal CBM policies. Quality, Reliability, Risk, Maintenance, and Safety Engineering (QR2MSE), $2013 \quad$ International Conference on: 700-706. http://dx.doi.org/ 10.1109/QR2MSE.2013.6625672

White, P. R., \& Locke, J. (2012). Performance of methods based on the FrFT for the detection of linear frequency modulated signals. IET Signals Processing, 6(5), 478-483. http://dx.doi.org/10.1049/iet-spr.2011.0189

White, P. R., \& Locke. J. (2012). Performance of methods based on the fractional Fourier transform for the detection of linear frequency modulated signals. IET Signal Processing, 6(5), 478-483. http://dx.doi.org/10.1049/iet-spr.2011.0189

\section{Copyrights}

Copyright for this article is retained by the author(s), with first publication rights granted to the journal.

This is an open-access article distributed under the terms and conditions of the Creative Commons Attribution license (http://creativecommons.org/licenses/by/3.0/). 